Hospital. There will also be a special course of instruction in infants' diseases at the Infants' Hospital, from September 3rd to 21st, a course in ophthalmology at the Central London Ophthalmic Hospital, from September 3rd to 29th, and a course in dermatology at St. John's Hospital for Diseases of the Skin, from September 17th to October 13th. Particulars of the courses may be obtained from the Secretary, Fellowship of Medicine, 1, Wimpole Street, W.1.

We announce in our Service notes this week the promotion of Surgeon Captain Arthur Reginald Bankart, C.V.O., R.N., now serving in H.M. Yacht Victoria and Albert, to Knight Commandership of the Royal Victorian Order. It was largely owing to Sir Reginald Bankart's personal influence that members of the British Medical Association attending the Annual Meeting at Portsmouth last month were able to visit the Royal Yacht.

\section{VARIETIES OF SMALL-POX.}

BY

JOHN C. McVAIL, M.D., LL.D., F.R.F.P.S.

THE extensive prevalence of mild small-pox in England last year and this year has naturally created much medical interest in the subject, and the purpose of this article is to review briefly the general position.

The subject is far from new. As pointed out by me in the Milroy lectures in 1919, Sydenham referred to smallpox without eruption. Wagstaffe, in the eighteenth century, said of small-pox that " there is one sort in which a nurse cannot kill, and another which even a physician can never cure." Jenner, in 1789 , seven years before his first vaccination, discussed the question whether a mild type of small-pox then existing might not be used for protective inoculation in preference to matter from the ordinary disease. Adams, of the London Small-pox Hospital, wrote on "pearl-pox" in 1806. About a century ago Professor Thomson of Edinburgh defended at much length the proposition that small-pox and chicken-pox are one disease, and, if I mistake not, Hebra was of the same opinion. In the eighteenth century names such as stonepox, horn-pox, water-pox, swine-pox were not uncommon, but probably referred mainly to modified second attacks of small-pox, which occasionally occurred at long intervals after a first attack, particularly, no doubt, if the first attack had been mild.

In recent years mild or modified small-pox has been much heard of in various parts of the world-in Trinidad, the United States, Canada, Australia, and South Africa. Some outbreaks have been the subject of careful and valuable investigation, as recorded in the Proceedings of the Royal Society of Medicine and in the Transactions of the Society of Tropical Medicine and Hygiene. Various names have been applied to such outbreaks in the countries where they have occurred-alastrim, Cuban itch, or, in South Africa, amaas, meaning milk-pox. In one instance such a cognomen was adopted to allay alarm in the country surrounding the invaded area, lest the alarm should prevent the bringing in of necessary supplies. But such nomenclature leads to confusion, and whatever is variolous should be so acknowledged, whether mild or severe.

In this country a most puzzling and anomalous outbreak in Suffolk and Norfolk was investigated in 1919 by Dr. Monckton Copeman, whose report, published by the Ministry of Health in its $1919-20$ volume, is of much interest. In a total of 31 cases only four were seriously ill, and the only death was that of a puny unvaccinated baby. The infection, it was supposed, might have had its origin in Egypt, and in the course of his early visits Dr. Copeman expressed the opinion that "as the disease gradually became more acclimatized" it would tend to "approach more nearly to the type of small-pox as ordinarily met with in this country." That, as it turned out, was the course of events; in the later cases the severity of the symptoms was markedly greater, and backache, for example, was added to the initial headache.

In that connexion a very important question is whether the exceedingly mild variola at present prevalent in Gloucester and elsewhere will continue to breed true-will be mild throughout the whole of its prevalence, or will, whether by degrees or suddenly, change its character and take on the classical appearances of small-pox so well known throughout the world. No certain answer can be given to the question. That close and competent observer, the late Dr. Franklin Parsons, related how " in one town in Lancashire there were outbreaks from two sources concurrently; one could be traced to the cases imported from America, of a mild type, while another was traceable to the infection which had come across from Paris via London, and that was severe." Indeed, the fatality rate from mild small-pox in this country is now even lower than in the prevalence of 1902-5, when the American type first became prominent on these shores. At that time fatality rates ran from about 1 per cent. to 4 or 5 or 6 per cent., including vaccinated and unvaccinated, but no large outbreak that I know of had so low a rate, say, as last half-year's two or three deaths in over 1,200 cases. In America we in this country have generally understood that mildness of type had become established so that the disease was in some places being regarded as endemic, and not worth troubling about. Now, however, the picture has suddenly changed. The United States Treasury Department, in its Public Health Report for the week ending June 22nd, 1923, has a note entitled "Increasing virulence of smallpox in the United States," and accompanies it by an important table of statistics, headed "Small-pox cases and deaths, and deaths per 100 cases, in 275 American and Canadian cities, 1920, 1921, and 1922." The population of the cities is not stated, but there is full information as to the number of cases and of deaths in each year for the total cities, and for ten or eleven "special cities." Canadian cities to the number of 29 are included in the 275 , because the data are contained in a statistical bulletin of the Metropolitan Life Insurance Company. Taking the cities as a whole, there were 30,328 cases in 1920 , 23,977 in 1921, and 8,709 in 1922 . The numbers, therefore, have decreased. But the fatality rate was 0.6 per cent. in $1920,1.1$ in 1921 , and 5.5 in 1922. In the special cities jointly, the fatality rate in 1920 was 0.2 per cent., in 19218.5 per cent., and in 1922 no less than 28.4 per cent. Of individual cities, Kansas and Denver were very hardly hit, the deaths being at the rates of 45 and 31 per cent. It is surmised that the outbreaks in these two towns may have had the same unknown origin, but whatever the sources it is clear that either the mild type has changed or a severe type has taken its place in these and other cities.

In no country can there be any certainty as to the future of small-pox, and the only prudent course is to be prepared against whatever may come. Vaccination, renewed as required, can prevent both severe and mild small-pox. The one form protects against the other, vaccination protects against both, and Chapin, when the mild form was spreading in America; pointed out, further, "that persons who have had the mild type are equally immune to vaccination." As to diagnosis, I would call attention to Dr. Wanklyn's article in the British Medical JournaL of July 21st (p. 106), and would also remind the profession that the Ministry of Health has appointed for each part of the country a consultant skilled in all that relates to variola. When doubt arises there should be no delay in calling in this aid. 